\title{
White Matter Hyperintensities Predict Cognitive Decline: A Community-Based Study
}

\author{
Xuemei Qi*, Huidong Tang*, Qi Luo, Bei Ding, Jie Chen, Peijing Cui, \\ Shengdi Chen, Huawei Ling, Jianfang Ma
}

ABSTRACT: Introduction: White matter hyperintensities (WMHs) were commonly seen in brain magnetic resonance imaging (MRI) of the elderly. Many studies found that WMHs were associated with cognitive decline and dementia. However, the association between WMHs in different brain regions and cognitive decline remains debated. Methods: We explored the association of the severity of WMHs and cognitive decline in 115 non-demented elderly ( $\geq 50$ years old) sampled from the Wuliqiao Community located in urban area of Shanghai. MRI scans were done during 2009-2011 at the beginning of the study. Severity of WMHs in different brain regions was scored by Improved Scheltens Scale and Cholinergic Pathways Hyperintensities Scale (CHIPS). Cognitive function was evaluated by Mini-Mental State Examination (MMSE) every 2 to 4 years during 2009-2018. Results: After adjusting for confounding factors including age, gender, education level, smoking status, alcohol consumption, depression, hypertension, diabetes, hyperlipidemia, brain infarcts, brain atrophy, apoE4 status, and baseline MMSE score, periventricular and subcortical WMH lesions as well as WMHs in cholinergic pathways were significantly associated with annual MMSE decline $(p<0.05)$, in which the severity of periventricular WMHs predicted a faster MMSE decline $(-0.187$ points/year, $95 \%$ confidence interval: $-0.349,-0.026, p=0.024)$. Conclusions: The severity of WMHs at baseline was associated with cognitive decline in the non-demented elderly over time. Interventions on WMH lesions may offer some benefits for cognitive deterioration.

RÉSUMÉ: Des hyper-signaux de la substance blanche prédicteurs du déclin cognitif : une étude menée dans une communauté locale. Introduction: Des hyper-signaux de la substance blanche (HSSB) peuvent généralement être observés lors d'examens d'imagerie par résonnance magnétique (IRM) effectués chez des personnes âgées. Plusieurs études ont également montré que les HSSB étaient associés au déclin cognitif et à la démence. Cela dit, le lien pouvant exister entre ces HSSB détectés dans diverses régions cérébrales et le déclin cognitif demeure sujet à débat. Méthodes: Nous avons décidé d'explorer l'association existant entre l'intensité des HSSB et le déclin cognitif chez 115 personnes âgées n'étant pas atteintes de démence ( $\geq 50$ ans). Ces personnes avaient été recrutées au sein du quartier de Wuliqiao situé dans le grand Shanghai. Signalons que ces examens d'IRM ont été effectués au début de cette étude entre 2009 et 2011. L'intensité des HSSB dans diverses régions cérébrales a été mesurée au moyen des échelles suivantes : la Improved Scheltens Scale et la Cholinergic Pathways Hyperintensities Scale (CHIPS). En ce qui concerne la fonction cognitive, elle a été évaluée à l'aide du test de Folstein (ou mini-mental state examination) tous les 2 à 4 ans entre 2009 et 2018. Résultats: Une fois la prise en compte d'un certain nombre de facteurs de confusion (l'âge, le sexe, le niveau de scolarité, le tabagisme, la consommation d'alcool, la dépression, l'hypertension, le diabète, l'hyperlipidémie, des accidents ischémiques cérébraux, une atrophie du cerveau, la situation de l'allèle 4 du gène ApoE et le score initial au test de Folstein), il est apparu que des lésions révélées par des hyper-signaux des régions péri-ventriculaire et sous-corticale, de même que des hyper-signaux détectés dans les voies cholinergiques, étaient nettement associés à des résultats en baisse au test de Folstein en cours d'année $(\mathrm{p}<0,05)$. Fait à noter, l'intensité des HSSB de la région péri-ventriculaire a aussi permis de prédire un déclin plus rapide des scores au test de Folstein (- 0,187 points/année, IC $95 \%$ : - 0,349 - 0,026; p =0,024). Conclusions: L'intensité des HSSB observée au début de cette étude a été associée au fil du temps au déclin cognitif de personnes âgées n'étant pas atteintes de démence. Il est donc possible que des interventions ciblant des lésions révélées par des HSSB puissent offrir certains bienfaits quand il est question de déclin cognitif.

Keywords: White matter diseases, Population-based study, Cognitive decline doi:10.1017/cjn.2019.47

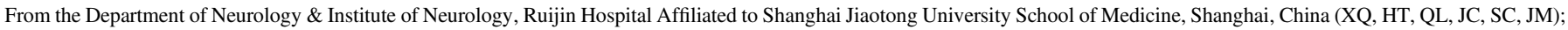

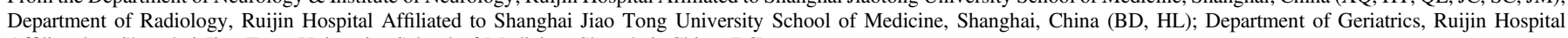
Affiliated to Shanghai Jiao Tong University School of Medicine, Shanghai, China (PC)

Received January 12, 2019. Final Revisions Submitted March 8, 2019. Date of Acceptance April 6, 2019.

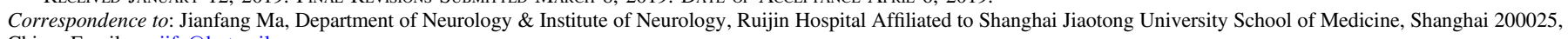
China. Email: majifa@hotmail.com

Huawei Ling, Department of Radiology, Ruijin Hospital Affiliated to Shanghai Jiao Tong University School of Medicine, Shanghai, China. Email: hwl1505@ sina.com

*Both authors contributed to the work equally. 


\section{INTRODUCTION}

White matter hyperintensities (WMHs) are common brain magnetic resonance imaging (MRI) changes in the elderly and play an important role in their quality of daily life. ${ }^{1}$ WMHs are usually located in basal ganglia, periventricular, subcortical or semioval center regions, caused by demyelination and axonal loss resulting from cerebral small vessel disease., ${ }^{2,3}$

Many studies suggested that WMHs were associated with cognitive impairment and dementia. ${ }^{4-7}$ The profile of WMHs related cognitive impairment usually involved executive dysfunction including attention or working memory, and information processing speed. ${ }^{3,4}$ Some studies suggested that the association of WMHs with cognitive impairment was due to sharing risk factors such as age, high blood pressure, smoking, cerebral infarction. ${ }^{3,8}$ Others argued that the site of WMHs lesions was the most important factor contributing to cognitive impairment, such as subcortical lesions or cholinergic pathways lesions. ${ }^{7,9,10}$

Therefore in this community-based study, we tried to investigate the association of the severity of WMHs in different areas, such as periventricular and subcortical brain regions as well as WMHs within cholinergic pathways, with longitudinal cognitive decline in 115 elderly participants ( $\geq 50$ years old) recruited from a population-based cohort with 9-year follow-up.

\section{MeTHODS \\ Participants}

This study population was recruited from a longitudinal Shanghai cohort on neurodegenerative diseases (dementia and Parkinson's disease) and sleep disorders established in 2009 and followed up in 2011, 2014 and 2018. ${ }^{11,12}$ The MRI sub-cohort was established in Wuliqiao Community, an urban community in Huangpu District. An MRI was done in 146 subjects ( $\geq 50$ years old) who were willing to enter the MRI study at baseline during 2009-2011. In this MRI sub-cohort, follow-up cognitive assessments were performed in 86 people in 2011, 107 people in 2014 and 67 people in 2018. Our follow-up ended in 15 December 2018, and the median follow-up time was 8 (95\% CI: 7.49-8.51) years. Among them, 31 subjects were excluded for the following reasons, dementia or cognitive impairment at baseline $(n=6)$, hearing impairment $(n=1)$, Parkinson's disease $(n=1)$, no follow-up Mini-Mental State Examination (MMSE scores $(n=11)$ ), suboptimal MRI quality $(n=1)$ and stroke lesions diameter $\geq 2 \mathrm{~cm}$ $(n=11)$. Finally, the data of 115 participants were included in our analysis. (See Figure 1.)

\section{Magnetic Resonance Imaging Scanning}

Cerebral MRI scanning was performed during 2009-2011 using a 1.5T GE Signa Horizon scanner (GE Medical Systems, Waukesha, WI). The scanning protocol included a series of T1-weighted,T2-weighted, FLAIR and diffusion weighted imaging (DWI) images. Sections were $5 \mathrm{~mm}$ thick.

WMHs were considered as hyperintensitense lesions presented on both FLAIR and T2-weighted images. We used Improved Scheltens Scale and Cholinergic Pathways Hyperintensities Scale (CHIPS) to access the severity of WMHs. For improved Scheltens Scale, WMHs adjacent to the ventricle were considered as the periventricular WMHs, otherwise as subcortical deep WMHs. ${ }^{4}$ Periventricular WMHs were scored

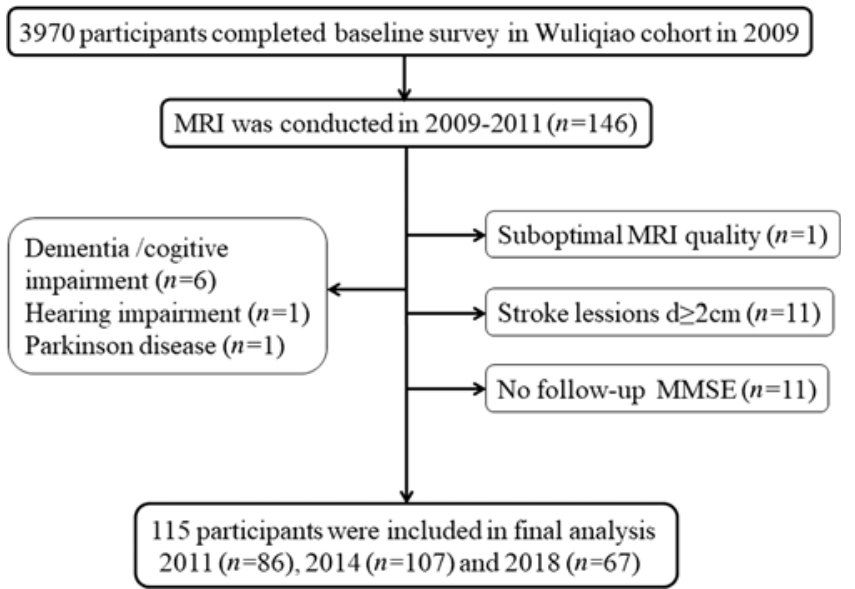

Figure 1: Flow chart of the study.

semi-quantitatively on a scale of 0-2 for $\mathrm{WMH}$ lesions located adjacent to the lateral ventricles and the frontal and occipital horns (0-none, 1-lesion $\leq 5 \mathrm{~mm}, 2$-lesions $>5 \mathrm{~mm}$ ). The total periventricular WMH score was added by the region-specific scores (range, 0-6). Subcortical deep WMHs were scored on a scale of 0-6 for lesions on frontal, parietal, occipital, and temporal lobes according to their diameter and number, as 0 (none), 1 (lesions $\leq 3 \mathrm{~mm}, \mathrm{n} \leq 5$ ), 2 (lesions $\leq 3 \mathrm{~mm}, \mathrm{n} \geq 6$ ), 3 (4 $\mathrm{mm} \leq$ lesions $\leq 10 \mathrm{~mm}, \mathrm{n} \leq 5), 4(4 \mathrm{~mm} \leq$ lesions $\leq 10 \mathrm{~mm}$, $\mathrm{n} \geq 6$ ), 5 (lesions $\geq 11 \mathrm{~mm}, \mathrm{n} \geq 1$ ) and 6 (large confluent lesions). Total subcortical deep WMH scales were calculated by the score of each cerebral lobe (range 0-24). ${ }^{13}$

The CHIPS was used to assess WMH lesions in cholinergic system. Briefly the cholinergic pathways were separated into 10 regions by using the major anatomical landmarks on four slices in the axial plane which span the third and lateral ventricles. The severity of WMH lesions was visually rated on a scale of $0-2$ for each region (0-none, 1 -mild $<50 \%$ involved, 2-moderate to severe $\geq 50 \%$ involved). Each slice was weighted according to the concentration of cholinergic fibers in different regions (weight from 1 to 4: low external capsule [weight $\times 4$ ], high external capsule [weight $\times 3$ ], corona radiate [weight $\times 2$ ], centrum semiovale [weight $\times 1]$ ). At last the maximum score of each hemisphere was 50 , with a total maximum score of 100 per scan.

To assess brain atrophy we calculated the ventricle-to-brain ratio (the mean of the biventricular width at the frontal and occipital horns divided by the corresponding brain width at the level of the caudate nuclei body) on T1-weighted images. ${ }^{4}$

Brain infarcts presented as hyperintense in T2-weighted images and hypointense in T1-weighted images and having a hyperintense rim surrounding the lesions in FLAIR sequences were categorized as present or absent in our study. ${ }^{14}$

Two independent experienced physicians rated all scans. If the scores disagreed on more than one point, we would hold a consensus reading. In other cases, scores were averaged. Interreader- and intrareader-intraclass correlation coefficients showed good agreement of reliability for periventricular and subcortical WMHs. Interreader- and intrareader-intraclass correlation coefficients for periventricular WMH scores were 0.931 and 0.872 , respectively. And the interreader- and intrareader-intraclass correlation coefficients for subcortical WMH scores were 0.881 and 0.937, respectively. Pearson's correlation coefficient between 
Table 1: Baseline characteristics for the study population

\begin{tabular}{|c|c|c|c|c|}
\hline Characteristics & Overall population $(n=3970)$ & Non-participants $(n=3855)$ & Participants $(n=115)$ & $P$ value \\
\hline Age, mean (SD) & $64.52(10.3)$ & $64.45(10.3)$ & $66.85(9.9)$ & 0.05 \\
\hline Women, n (\%) & $2672(67.3)$ & $2589(67.2)$ & $83(72.2)$ & 0.53 \\
\hline \multicolumn{5}{|c|}{ Education, n (\%) } \\
\hline Elementary or middle school & $2469(63.2)$ & $2392(63.1)$ & $77(67.0)$ & 0.81 \\
\hline High school & $1012(25.9)$ & $988(26.0)$ & $24(20.9)$ & \\
\hline University & $427(10.9)$ & $413(10.9)$ & $14(12.2)$ & \\
\hline Alcohol drinker, n (\%) & $789(19.9)$ & $775(20.1)$ & $14(12.2)$ & 0.11 \\
\hline Ever smoking, n (\%) & $483(12.2)$ & $463(12.0)$ & $20(17.4)$ & 0.22 \\
\hline Hypertension, n (\%) & $2652(66.8)$ & $2558(66.4)$ & $94(81.7)$ & $<0.01$ \\
\hline Diabetes, n (\%) & 1149 (28.9) & $1105(28.7)$ & $44(38.3)$ & 0.08 \\
\hline Hyperlipidemia, n (\%) & $1478(37.2)$ & $1410(36.6)$ & $68(59.1)$ & $<0.001$ \\
\hline Depression, n (\%) & $380(9.6)$ & $364(9.4)$ & $16(13.9)$ & 0.28 \\
\hline MMSE score, mean (SD) & $27.27(3.2)$ & $27.28(3.2)$ & $27.17(2.7)$ & 0.88 \\
\hline
\end{tabular}

Abbreviations: $\mathrm{SD}=$ standard deviation, $\mathrm{MMSE}=$ Mini-Mental Examination .

Education level was available for 3908 subjects.

periventricular and subcortical WMHs was 0.786 . Of all subjects, only $6.07 \%$ had no signs of WMHs, while $8.70 \%$ had no periventricular WMLs and $11.30 \%$ were free of subcortical WMHs. And $86.09 \%$ of all subjects had both periventricular and subcortical WMHs. WMHs scoring in cholinergic system also showed very good agreement for both interreader $(\mathrm{ICC}=0.896$ ) and intrareader ( $\mathrm{ICC}=0.941$ ) reliability. Of all subjects, only $6.96 \%$ had no signs of WMHs in cholinergic system.

\section{Assessment of Cognitive Function and Other Measurements}

Global cognitive function at baseline and follow-up was assessed by MMSE for all participants. Cognitive impairment was defined based on MMSE scores with different cut-offs according to education level. ${ }^{15}$ In addition to cognitive assessment, we conducted face-to-face interviews to collect subjects' demographic information, alcohol consumption, smoking status, medical history (hypertension, diabetes mellitus, hyperlipidemia, other co-morbidities) and psychiatric history (e.g. depression and mental illness). Genomic DNA was extracted by the standard method. ApoE $\varepsilon 4$ genotyping was amplified by polymerase chain reaction (PCR) and performed as previously described. ${ }^{15}$

\section{Ethics Statement}

This study was approved by the Research Ethics Committee, Ruijin Hospital affiliated to Shanghai Jiao Tong University School of Medicine, China. All participants signed written informed consents.

\section{Statistical Analysis}

We used $t$-test and one way-ANOVA for continuous variables normally distributed, Wilcoxon-Mann-Whitney test for continuous variables without normal distribution and chi-square test for categorical variables. Liner mixed-effects models for repeated measure were used to analyze the association of the severity of
WMHs with MMSE scores. WMH scores were square root transformed before entering the liner mixed-effects model. The model included WMH scores at baseline, follow-up time, and the interaction between WMH scores and follow-up time. The estimated effect of the interaction term reflects the impact of the WMH scores on the annual change of MMSE score. ${ }^{16}$ Model 1 was adjusted for age, sex, and education level. Model 2 was further adjusted for smoking, alcohol, depression, hypertension, diabetes, hyperlipidemia, brain infarcts, brain atrophy, apoE $\varepsilon 4$ status, and baseline MMSE scores. The relation between subcortical WMH lesions and annual MMSE decline was further conditional on the severity of periventricular WMHs and vice versa. $^{17}$ All analysis was performed by SPSS 19.0 statistic software (SPSS Inc., Chicago, IL, USA).

\section{RESULTS}

\section{Baseline Characteristics of the MRI Study Population}

The mean age of the 115 enrolled participants of MRI cohort was 66.85 years. About $72.2 \%$ were women and $12.2 \%$ obtained university degree. There was no difference with regard to demographic characteristics, education, smoking and alcohol consumption, depression, diabetes, and baseline MMSE score among MRI participants, nonMRI participants, and the whole cohort $(p>0.05)$. However, our MRI study participants had a higher proportion of hypertension (81.7\% vs. $66.8 \%$ or $66.4 \%, p<0.01)$ and hyperlipidemia $(59.1 \%$ vs. $36.6 \%$ or $37.2 \%, p<0.001$ ). (Table 1 ).

When comparing participants who had all follow-up visits with others who missed at least one follow-up visits, we found that subjects who did not participate at least one follow-up visit had a higher proportion of small cerebral infarcts (34.8\% vs. $15.2 \%, p=0.02$ ), without any differences in demographics features, education level, living habits, hypertension, diabetes, hyperlipidemia, depression, baseline MMSE score, severity of WMH lesions, and brain atrophy (Table 2). 
Table 2: Baseline characteristics of participants by follow-up visits

\begin{tabular}{|c|c|c|c|}
\hline Characteristics & Participate all the visits $(n=46)$ & $\begin{array}{l}\text { At least one visit did not participate } \\
\qquad(n=69)\end{array}$ & $P$ value \\
\hline Age, mean (SD) & $65.59 \pm 9.6$ & $67.722 \pm 10.1$ & 0.258 \\
\hline Women, n (\%) & $37 / 46(80.4)$ & $46 / 69(66.7)$ & 0.138 \\
\hline \multicolumn{4}{|c|}{ Education, n (\%) } \\
\hline Elementary or middle school & $33 / 46(71.7)$ & $42 / 69(60.9)$ & 0.483 \\
\hline High school & $8 / 46(17.4)$ & $16 / 69(23.2)$ & \\
\hline University & $5 / 46(10.9)$ & $11 / 69(15.9)$ & \\
\hline Alcohol drinker, n (\%) & $5 / 46(10.9)$ & $10 / 69(14.5)$ & 0.572 \\
\hline Ever smoking, n (\%) & $10 / 46(21.7)$ & $11 / 69(15.9)$ & 0.431 \\
\hline Hypertension, n (\%) & $34 / 46(73.9)$ & $52 / 68(75.4)$ & 0.861 \\
\hline Diabetes, n (\%) & $14 / 46(30.4)$ & $30 / 69(43.5)$ & 0.159 \\
\hline Hyperlipidemia, n (\%) & $31 / 46(67.4)$ & $39 / 68(56.5)$ & 0.242 \\
\hline Depression, n (\%) & $13 / 46(28.3)$ & $20 / 69(29.0)$ & 0.933 \\
\hline ApoE4 status, n (\%) & $3 / 46(6.5)$ & $9 / 69(13.0)$ & 0.418 \\
\hline MMSE score at baseline, mean (SD) & $27.13 \pm 2.3$ & $27.13 \pm 3.0$ & 0.999 \\
\hline \multicolumn{4}{|c|}{ MRI markers } \\
\hline Scheltens Scale, median (IQR) & $4.0(2.0 ; 6.5)$ & $6.0(3.0 ; 10.5)$ & 0.053 \\
\hline CHIP Scale, median (IQR) & $13.0(9.5 ; 21.5)$ & $16.0(10.5 ; 26.0)$ & 0.164 \\
\hline Brian infarct, n (\%) & $7 / 46(15.2)$ & $24 / 69(34.8)$ & 0.021 \\
\hline Ventricle/brain ratio, mean (SD) & $0.36 \pm 0.02$ & $0.37 \pm 0.02$ & 0.188 \\
\hline
\end{tabular}

$\mathrm{SD}=$ standard deviation, MMSE = Mini-Mental Examination, $\mathrm{IQR}=$ interquartile range, $\mathrm{CHIP}=$ Cholinergic Pathways Hyperintensities.

Table 3: Estimates of effects of WMH scales on annual change in the MMSE score

\begin{tabular}{c|c|c}
\hline WMH scales & Model 1 & Model 2 \\
\hline \multicolumn{3}{|c|}{ Scheltens Scales } \\
\hline Total WMH & $-0.168(-0.275,-0.061) 0.002$ & $-0.134(-0.231,-0.037) 0.008$ \\
\hline Periventricular WMH $^{\mathrm{a}}$ & $-0.242(-0.419,-0.066) 0.008$ & $-0.187(-0.349,-0.026) 0.024$ \\
\hline Subcortical WMH $^{\mathrm{b}}$ & $-0.179(-0.297,-0.062) 0.003$ & $-0.139(-0.245,-0.033) 0.011$ \\
\hline CHIP Scales & $-0.107(-0.177,-0.036) 0.003$ & $-0.092(-0.156,-0.028) 0.005$ \\
\hline
\end{tabular}

$\mathrm{CI}=$ confidence interval; $\mathrm{WMH}=$ white matter hyperintensities; CHIP Scales = Cholinergic Pathways Hyperintensities Scale; MMSE $=$ Mini-Mental State Examination.

Model 1 was adjusted for age, sex and education; Model 2 was additionally adjusted for smoking, alcohol, depression, hypertension, diabetes, hyperlipidemia, apoE4 status, brain infarcts, brain atrophy and baseline MMSE score in model 1.

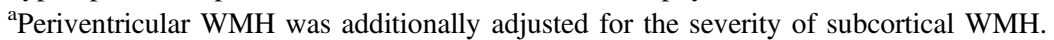

${ }^{\mathrm{b}}$ Subcortical WMH was additionally adjusted for the severity of periventricular WMH.

\section{Baseline WMH Severity and Annual MMSE Change}

After adjusting for age, sex, and education, the MMSE score declined by $0.224(95 \% \mathrm{CI}:-0.346,-0.102)$ points every year during the follow-up period. The overall WMHs severity at baseline was associated with annual MMSE decline (rate $=-0.168 /$ year, 95\% CI: $-0.0275,-0.061, p=0.002)($ Table 3, Model 1). This association remained statistically significant after adjusting for smoking status, alcohol consumption, depression, hypertension, diabetes, hyperlipidemia, brain infarcts, brain atrophy, apoE $\varepsilon 4$ status, and baseline MMSE score (rate $=-0.134$ / year, 95\% CI: $-0.231,-0.037, p=0.008$ ) (Table 3, Model 2).

After adjusting for age, sex, education, and the severity of subcortical WMHs, periventricular WMH severity at baseline was found associated with annual MMSE decline (rate $=-0.242$ / year, 95\% CI: $-0.419,-0.066, p=0.008$ ) (Table 3, Model 1). This association remained significant when further adjusted for smoking status, alcohol consumption, depression, hypertension, diabetes, hyperlipidemia, apoE $\varepsilon 4$ status, brain infarcts, brain 
atrophy, and baseline MMSE score (rate $=-0.187 /$ year, $95 \%$ CI: $-0.349,-0.0226, p=0.029$ ) (Table 3, Model 2).

Similarly, subcortical WMH severity at baseline was also found to be associated with annual MMSE decline after adjusting for age, sex, education, and the severity of periventricular WMH lesions (rate $=-0.179 /$ year, 95\% CI: $-0.297,-0.062, p=0.003$ ) (Table 3, Model 1) and other confounders as discussed earlier (rate $=-0.139 /$ year, 95\% CI: $-0.245,-0.033, \mathrm{p}=0.011$ ) (Table 3, Model 2).

Our results also showed that the severity of WMH within cholinergic pathways at baseline was linked to cognitive decline after adjusting for age, sex, and education (rate $=-0.107 /$ year, 95\% CI: $-0.177,-0.036, p=0.003$ ) (Table 3, Model 1). The association was still statistically significant after adjusting for other potential confounder as discussed earlier (rate $=-0.092 /$ year, 95\% CI: $-0.156,-0.028, p=0.005$ ) (Table 3, Model 2).

\section{DISCUSSION}

In the present study, the MMSE score declined by 0.224 points per year after adjusting for age, sex, and education level, which was in accordance with findings in previous studies. ${ }^{17,18}$ We found that the severity of WMH lesions at baseline was associated with annual MMSE decline in the non-demented Shanghai elderly population after a 9-year follow-up. Overall WMH severity, WMH lesions either in periventricular and subcortical regions evaluated by Scheltens Scale or WMH lesions in cholinergic pathways evaluated by CHIP were all significantly associated with greater annual MMSE decline after adjusting for potential confounders, apoE $\varepsilon 4$ status, and baseline MMSE score. These findings were consistent with other prospective studies. ${ }^{3,4,17}$ In the Rotterdam Scan Study, baseline WMH lesions in the periventricular region were associated with the decline on the MMSE performance. ${ }^{17}$ Silbert et al. found that the subcortical WMH volume change was paralleled with cognitive decline in the cognitively intact elderly. In the Leukoaraiosis and Disability Study (LADIS) study, the progression of WMH lesions rather than the severity at baseline was better correlated with cognitive decline. ${ }^{19,20}$

Regarding the strong association between apoE $\varepsilon 4$ allele and cognitive decline ${ }^{21,22}$, however, these studies did not investigate the interaction of apoE $\varepsilon 4$ allele with severity of WMH lesions. Recently, Rui et al. found that larger volume of WMH lesions at baseline was associated with faster MMSE score decline after a 9-year follow-up and apoE $\varepsilon 4$ allele could magnify the effect of WMH lesions on cognitive decline compared with non-carriers. ${ }^{16}$ Our results showed that the overall WMHs severity predicted 0.134 points decline per year after adjusting for all potential confounders including apoE $\varepsilon 4$, similarly to 0.112 points decline in Rui's study.

Several pathophysiological pathways are suggested to be involved in the association between WMH lesions and cognitive decline in aging people. One possible mechanism is the disruption of the fronto-subcortical or cortical association fibers of cognitive process. ${ }^{23}$ Vascular architecture in periventricular region is more vulnerable to ischemic damage than other white matter areas. In addition, the periventricular area has a high density of long fibers connecting the cortex with subcortical nuclei as well as other distant brain structures, whereas the subcortical area has high density of short-looped U fibers which connect the adjacent cortical areas. ${ }^{24-26}$ Most of fibers projecting to cortical areas from the nucleus basalis are unmyelinated, which are vulnerable to vascular lesions in these areas. ${ }^{27}$ Another mechanism is that WMHs may interfere with the neural circuits of cholinergic pathway, the vital neurotransmitter of cognitive network. ${ }^{7,9,28}$ Cross-sectional studies revealed that WMHs in cholinergic pathways were correlated with cognitive performance in Alzheimer's disease (AD) patients as well as normal elderly. ${ }^{7,9}$ Prospective study found that the disruption of cholinergic pathways by acute ischemic stroke contributed to newly developed dementia. ${ }^{29}$ Our study also confirmed that WMHs in the cholinergic pathways at baseline predicted annual cognitive decline in the normal elderly by MMSE scores.

Strengths in our study include a prospective community-based study population with the longitudinal assessment of cognitive function. However, there are still some limitations. First, a small sample size decreased the power to reveal the influences of WMH lesions on cognitive decline. Second, we only accessed WMH lesions at baseline and did not assess the progression of WMH lesions over time. So we cannot be sure that the MMSE decline might be attributed to WMH progression or other features such as silent infarction. Thirdly, we were not able to perform more detailed neuropsychological tests other than MMSE to assess the cognitive function. Although MMSE is widely used in global cognitive function screening in population-based study, it is less sensitive than other scales to detect MCI patients. Finally, our MRI sub-cohort recruited more hypertension and hyperlipidemia people than the whole cohort, and these two factors were associated with WMHs severity as well as cognitive decline. ${ }^{16,30}$ Although we adjusted these two confounders in the statistical model, we could not rule out the possibility of recruitment bias and its effects on the relation between the severity of WMH lesions and cognitive decline.

Despite these limitations, this population-based prospective study provided evidences indicating that WMHs burden at baseline was associated with cognitive decline in Shanghai elderly. Overall WMHs, perivascular or subcortical WMHs, as well as WMHs in cholinergic pathways were all associated with longitudinal cognitive decline. It is important to detect early and thus prevent the progression of WMH lesions to delay the processes of cognitive deterioration and even dementia in the future.

\section{ACKNowledgements}

We thank all the doctors from Wuliqiao Medical Center for their support to our epidemiology study.

\section{Funding}

This study was supported by grants from National Natural Science Fund $(81571103,81501086)$, the National Key R\&D Program of China (2016YFC1306000).

\section{Disclosures}

The authors have no conflict of interests to declare.

\section{Statement of Authorship}

XMQ and HDT collected the demographic data and assessed the WMHs, performed the statistical analysis, and drafted the 
manuscript. JC and QL collected demographic data and followup assessment. PJC performed the apoE genotype. BD doublechecked the WMHs assessment. SDC revised the manuscript. HWL trained XMQ and HDT for WMH assessment, designed the study and revised the manuscript. JFM designed the study, double-checked the statistical analysis, and revised the manuscript.

\section{REFERENCES}

1. Longstreth WT, Jr., Diehr PH, Yee LM, Newman AB, Beauchamp NJ. Brain imaging findings in elderly adults and years of life, healthy life, and able life over the ensuing 16 years: the Cardiovascular Health Study. J Am Geriatr Soc. 2014;62: $1838-43$.

2. van Dijk EJ, Prins ND, Vermeer SE, Koudstaal PJ, Breteler MM. Frequency of white matter lesions and silent lacunar infarcts. J Neural Trans Suppl. 2002;62:25-39.

3. Prins ND, Scheltens P. White matter hyperintensities, cognitive impairment and dementia: an update. Nat Rev Neurol. 2015; $11: 157-65$

4. de Groot JC, de Leeuw FE, Oudkerk M, et al. Cerebral white matter lesions and cognitive function: the Rotterdam Scan Study. Ann Neurol. 2000;47:145-51.

5. Mungas D, Jagust WJ, Reed BR, et al. MRI predictors of cognition in subcortical ischemic vascular disease and Alzheimer's disease. Neurology. 2001;57:2229-35.

6. Kramer JH, Reed BR, Mungas D, Weiner MW, Chui HC. Executive dysfunction in subcortical ischemic vascular disease. J Neurol Neurosurg Psychiatr. 2002;72:217-20.

7. Ishikawa $\mathrm{H}$, Meguro $\mathrm{K}$, Ishii $\mathrm{H}$, Tanaka N, Yamaguchi S. Silent infarction or white matter hyperintensity and impaired attention task scores in a nondemented population: the Osaki-Tajiri Project. J Stroke Cerebrovasc Dis. 2012;21:275-82.

8. Wardlaw JM, Smith EE, Biessels GJ, et al. Neuroimaging standards for research into small vessel disease and its contribution to ageing and neurodegeneration. Lancet Neurol. 2013;12: 822-38.

9. Bocti C, Swartz RH, Gao FQ, Sahlas DJ, Behl P, Black SE. A new visual rating scale to assess strategic white matter hyperintensities within cholinergic pathways in dementia. Stroke 2005;36:2126-31.

10. Engelhardt E, Moreira DM, Laks J. Vascular dementia and the cholinergic pathways. Dementia Neuropsychol. 2007;1:2-9.

11. Tang HD, Zhou Y, Gao X, et al. Prevalence and risk factor of cognitive impairment were different between urban and rural population: a community-based study. J Alzheimer's Dis. 2016;49:917-25.

12. Ma JF, Qiao Y, Gao X, et al. A community-based study of risk factors for probable rapid eye movement sleep behavior disorder. Sleep Med. 2017;30:71-6.

13. Scheltens P, Barkhof F, Leys D, et al. A semiquantative rating scale for the assessment of signal hyperintensities on magnetic resonance imaging. J Neurol Sci. 1993;114:7-12.
14. Riba-Llena I, Koek M, Verhaaren BF, et al. Small cortical infarcts: prevalence, determinants, and cognitive correlates in the general population. Int J Stroke 2015;10(Suppl A100):18-24.

15. Xia XS, Li X, Wang L, Wang JZ, Ma JP, Wu CJ. Supplementation of folic acid and vitamin $\mathrm{B}(1)(2)$ reduces plasma levels of asymmetric dimethylarginine in patients with acute ischemic stroke. J Clin Neurosci. 2014;21:1586-90.

16. Wang R, Fratiglioni L, Kalpouzos G, et al. Mixed brain lesions mediate the association between cardiovascular risk burden and cognitive decline in old age: a population-based study. Alzheimer's Dementia. 2017;13:247-56.

17. De Groot JC, De Leeuw FE, Oudkerk M, et al. Periventricular cerebral white matter lesions predict rate of cognitive decline. Ann Neurol. 2002;52:335-41.

18. Bernier PJ, Gourdeau C, Carmichael PH, et al. Validation and diagnostic accuracy of predictive curves for age-associated longitudinal cognitive decline in older adults. CMAJ: Can Med Assoc J. 2017;189:E1472-80.

19. Silbert LC, Nelson C, Howieson DB, Moore MM, Kaye JA. Impact of white matter hyperintensity volume progression on rate of cognitive and motor decline. Neurology. 2008;71:108-13.

20. Schmidt $\mathrm{R}$, Berghold A, Jokinen $\mathrm{H}$, et al. White matter lesion progression in LADIS: frequency, clinical effects, and sample size calculations. Stroke. 2012;43:2643-47.

21. Liu CC, Liu CC, Kanekiyo T, Xu H, Bu G. Apolipoprotein E and Alzheimer disease: risk, mechanisms and therapy. Nat Rev Neurol. 2013;9:106-18.

22. Morris JC, Roe CM, Xiong C, et al. APOE predicts amyloid-beta but not tau Alzheimer pathology in cognitively normal aging. Ann Neurol. 2010;67:122-31.

23. Tullberg M, Fletcher E, DeCarli C, et al. White matter lesions impair frontal lobe function regardless of their location. Neurology. 2004;63:246-53.

24. Tuladhar AM, Reid AT, Shumskaya E, et al. Relationship between white matter hyperintensities, cortical thickness, and cognition. Stroke. 2015;46:425-32.

25. Pantoni L, Garcia JH. Pathogenesis of leukoaraiosis: a review. Stroke. 1997;28:652-59.

26. Bolandzadeh N, Liu-Ambrose $\mathrm{T}$, Aizenstein $\mathrm{H}$, et al. Pathways linking regional hyperintensities in the brain and slower gait. NeuroImage. 2014;99:7-13.

27. Selden NR, Gitelman DR, Salamon-Murayama N, Parrish TB, Mesulam MM. Trajectories of cholinergic pathways within the cerebral hemispheres of the human brain. Brain: J Neurol. 1998;121(Pt 12):2249-57.

28. Roman GC, Kalaria RN. Vascular determinants of cholinergic deficits in Alzheimer disease and vascular dementia. Neurobiol Aging. 2006;27:1769-85.

29. Lim JS, Kim N, Jang MU, et al. Cortical hubs and subcortical cholinergic pathways as neural substrates of poststroke dementia. Stroke. 2014;45:1069-76.

30. Qiu LN, Wang L, Li X, Han RF, Xia XS, Liu J. Predictive value of high residual platelet reactivity by flow cytometry for outcomes of ischemic stroke patients on clopidogrel therapy. J Stroke Cerebrovasc Dis. 2015;24:1145-52. 